\title{
COGNIÇÃO E INDICADORES DE SINTOMAS DEPRESSIVOS EM PESSOAS IDOSAS
}

\section{COGNITION AND INDICATORS OF DEPRESSIVE SYMPTOMS IN ELDERLY PEOPLE}

Thais da Silva-Ferreira ${ }^{1}$, Jeniffer Ferreira-Costa ${ }^{2}$, Glacielle Glayce Bento Marques $^{3}$, Daniel Bartholomeu ${ }^{4}$, José Maria Montiel ${ }^{5}$

\section{RESUMO}

Este estudo tem por objetivo identificar vulnerabilidades na autonomia e independência que imbricados nos aspectos cognitivos e de sintomas depressivos em pessoas idosas A amostra foi composta por 20 participantes de ambos os sexos, com idade igual ou superior a 62 anos. Os instrumentos utilizados foram o Mini Exame do Estado Mental (MEEM), a Escala de depressão geriátrica (EDG) abreviada com 15 itens e entrevistas semi-estruturadas. Os resultados demonstraram que todos os indivíduos possuíam percepções negativas sobre comprometimentos na autonomia e independência, também foi aferido que os idosos apresentaram diminuição nas atividades cotidianas. Dentre os participantes $55 \%$ apresentaram comprometimentos nos indicadores cognitivos e $30 \%$ a presença de sintomas depressivos. A quantidade de sintomas depressivos nos indivíduos que apresentaram comprometimento cognitivo foi $30 \%$ maior. Conclui-se que a presença de alterações cognitivas se apresentou como um preditor para presença de sintomas depressivos. A presença de alterações cognitivas e sintomalogia depressiva apresentaram correlação com a vulnerabilidade sobre a autonomia e independência de pessoas idosas, sendo estes, aspectos com influência direta na qualidade de vida e no bem-estar da população longeva.

Palavras-chave: Envelhecimento. Qualidade de vida. Independência.

\section{ABSTRACT}

The present study aims to identify the vulnerabilities in the autonomy and independence that are interweave on cognitive and depressive symptoms in elderly people. The sample was comprised by 20 participants of both sexes, aged 62 or over. The participants were assessed by the Mini Mental State Examination (MMSE), the Geriatric Depression Scale (EDG) abbreviated with 15 items and semi-structured interviews. The results showed that all individuals had negative perceptions about impairments in autonomy and independence, it was also verified that the elderly showed a decrease in daily activities. Among the participants, 55\% had impaired cognitive indicators and $30 \%$ had depressive symptoms. The amount of depressive symptoms in individuals who presented cognitive impairment was 30\% higher. We concluded that the presence of cognitive alterations was a predictor of the greater presence of depressive symptoms. The presence of cognitive alterations and depressive symptoms were correlated with vulnerability on the autonomy and independence of elderly people, which are aspects with direct influence on the quality of life and well-being of the long-lived population.
Graduanda do curso de Psicologia pela Universidade São Judas Tadeu - São Paulo, SP. E-mail: thais.sil.fe@hotmail.com

Graduanda do curso de Psicologia pela Universidade São Judas Tadeu - São Paulo, SP.

Mestranda em Ciências do Envelhecimento pela Universidade São Judas Tadeu, São Paulo, SP.

${ }^{4}$ Doutor em Psicologia, docente na UniAnchieta - Jundiaí, SP.

${ }^{5}$ Doutor em Psicologia, docente na Universidade São Judas Tadeu - São Paulo, SP.

Keywords: Aging. Quality of life. Independence. 


\section{INTRODUÇÄO}

No processo de desenvolvimento humano o organismo sofre mudanças e transformações. Com a chegada da ancianidade a diminuição na capacidade homeostática aumenta a vulnerabilidade às agressões externas e internas ao organismo. ${ }^{1} \mathrm{O}$ âmbito neurológico ${ }^{2}$ e psicossocial são amplamente transpassados por modificações que interferem diretamente na qualidade de vida da população idosa. ${ }^{3}$

Diante disso, indivíduos idosos necessitam de mecanismos adaptativos para atender às demandas requeridas nessa fase de vida. ${ }^{4} \mathrm{~A}$ capacidade adaptativa envolve estratégias internas e externas de seleção, otimização e compensação de perdas. ${ }^{5}$ Parte desta capacidade adaptativa é baseada nos recursos de autonomia e independência, com influência na saúde, bem-estar e qualidade de vida da população longeva. ${ }^{6-7}$

O Instituto Brasileiro de Geografia e Estatística ${ }^{8}$ aponta na Projeção da População o envelhecimento populacional crescente. É previsto que no ano de 2060, pessoas com mais de 65 anos componham aproximadamente $25,5 \%$ do total da população brasileira. Além disso, há o aumento da expectativa de vida, em 2010 a média de idade era de 73,86 anos, já em 2060 há previsão que a expectativa se eleve para 81,05 anos. Na Pesquisa Nacional de Saúde também realizada pelo Instituto Brasileiro de Geografia e Estatística, ${ }^{9}$ o maior acometimento do transtorno depressivo foi encontrado na faixa etária dos 60 aos 64 anos, com a prevalência de $11,1 \%$ neste grupo.

O transtorno depressivo possui forte influência hereditária, a natureza genética da depressão interage diretamente com fatores ambientais, devido ao caráter modificável deste, intervenções que gerem alterações no meio são importantes ferramentas na diminuição do risco de desenvolvimento do transtorno. ${ }^{2}$ Maiores níveis de apoio social e de reserva cognitiva estão relacionados com menores indicadores de sintomalogia depressiva em idosos. Ademais, o suporte social tem relação direta com o estado de saúde e percepções positivas sobre a vida, diminuindo sentimentos negativos que geralmente estão associados ao envelhecimento como a solidão e sentimento de insegurança. ${ }^{10}$

Seguindo os apontamentos da Organização Mundial de Saúde, ${ }^{11}$ o conceito saúde pode ser definido como "um estado de bem-estar físico, mental e social e não meramente a ausência de doença e enfermidade/doença". Essa definição além de ampliar o conceito de saúde, enfatiza o caráter subjetivo e multidimensional de critérios a serem considerados em avaliações das áreas da saúde. 
Essa ampliação conceitual coloca em foco a preservação e reconhecimento da autonomia e independência da população idosa. A autonomia é definida como o exercício e a noção de liberdade individual nas tomadas de decisões, baseia-se em diversas habilidades que permitem compreender a realidade, avaliar alternativas, definir metas e comunicar-se assertivamente no âmbito interpessoal. Já o conceito de independência, envolve o aspecto funcional do sujeito, expresso na capacidade de realizar atividades cotidianas e de autocuidado, ${ }^{12}$ Respeitar a autonomia do outro é considerar suas opiniões, objetivos e escolhas, sem obstruir o repertório de decisões e ações desse determinado sujeito.

Ambas as aptidões de autonomia e independência estão amparadas na cognição. Com o envelhecimento determinadas habilidades cognitivas comumente passam por modificações, algumas delas entram em declínio como a orientação topográfica, memória recente, capacidade de resolução de problemas, capacidade motora e o processamento de informações; enquanto outras aptidões declinam levemente, sendo elas as habilidades de compreensão, nomeação, fluência verbal, memória operativa, atenção complexa e funções executivas; enquanto a memória imediata e de longo prazo, atenção simples e o raciocínio prático, apresentam declínios variáveis entre indivíduos. ${ }^{13}$

O transtorno depressivo também sensibiliza determinados constructos cognitivos, estudos voltados às especificidades da relação entre cognição e depressão na população idosa indicam comprometimentos nos seguintes domínios: função executiva, ${ }^{14-15}$ memória episódica, memória de trabalho, linguagem, ${ }^{14,16-17}$ atenção, pensamento abstrato, ${ }^{15}$ aprendizagem ${ }^{17-18}$ e na velocidade de processamento. ${ }^{14}$

O transtorno depressivo desenvolvido tardiamente apresenta presença elevada de sintomas somáticos e/ou hipocondríacos, além disso, indivíduos com depressão geriátrica possuem menores taxas de resposta a tratamentos e de antecedentes genéticos de depressão. O tratamento adequado do transtorno depressivo ocasiona recuperação e proteção dos âmbitos cognitivos afetados, o que influencia positivamente não apenas a qualidade de vida dos indivíduos idosos, mas também serve como um estimulante para a família e médicos no tratamento de possíveis comorbidades. ${ }^{19}$

Tais assuntos demonstram a importância de pesquisas tencionadas sobre o âmbito do envelhecimento humano. Considerando os apontamentos aqui reunidos, faz-se necessário investigar as influências externas e internas que impactem na qualidade de vida e saúde da população longeva, favorecendo o entendimento do sujeito idoso como um ser 
dinâmico. Os constructos de autonomia e independência estão diretamente associados a esse dinamismo humano, logo compreender fatores específicos cognitivos e de humor que potencialmente estão relacionados a tais capacidades torna-se relevante. Portanto, este estudo possui como objetivo indicar que aspectos cognitivos e de sintomas depressivos tendem a estar relacionados ou imbricados em pessoas idosas.

\section{MATERIAIS E METODOS}

A presente pesquisa possui caráter qualitativo com análises descritivas e inferenciais, com dados coletados em uma amostra de idosos da cidade de São Paulo, SP. A amostra foi composta por 20 participantes, destes 6 do sexo masculino (30\%) e 14 do sexo feminino (70\%), com idades iguais ou superiores a 62 anos. A média de idade aferida foi igual a 69 anos. Dentre os participantes 03 possuíam de 1 a 4 anos de estudo, 04 possuíam de 9 a 11 anos de estudo e 13 indivíduos possuíam mais que 11 anos de estudo.

Os instrumentos utilizados na coleta de dados foram o Mini exame do estado mental (MEEM), Escala de Depressão Geriátrica (EDG) e entrevista semiestruturada.

Mini exame do estado mental (MEEM), criado por Folstein, Folstein e $\mathrm{McHugh}^{20} \mathrm{em}$ 1975, é um teste de rastreio do desempenho cognitivo que possibilita a investigação dos domínios de orientação temporal, orientação espacial, registro de memória, atenção, cálculo, memória de evocação e linguagem. O escore total é de 30 pontos, a nota de corte indicada é orientada pela escolaridade do indivíduo, correlato a 20 pontos para analfabetos, 25 pontos para indivíduos com 1 a 4 anos de estudo, 26 pontos para 5 a 8 anos de estudo, 28 pontos para 9 a 11 anos e 29 pontos para aqueles com mais de 11 anos de estudo. ${ }^{21} \mathrm{O}$ MEEM possui validação e adaptação para a população brasileira. Seu uso é indicado tanto no contexto clinico como em pesquisas. ${ }^{22-23}$

Escala de Depressão Geriátrica (EDG), criada por Yesavage et al..$^{24}$ em 1983 é um instrumento que possibilita o rastreio de sintomas depressivos na população idosa. É um questionário composto por perguntas claras e concisas que demandam respostas diretas de "sim" ou "não". No presente estudo a versão reduzida com 15 itens foi utilizada. A pontuação máxima equivale a 15 pontos, onde 0 é a ausência de sintomas depressivos e 15 corresponde a presença máxima dos sintomas depressivos avaliados. A nota de corte utilizada foi de 5 pontos, ou seja, pontuações iguais ou superiores a 5 indicam a presença de sintomas depressivos nos idosos. ${ }^{25} \mathrm{O}$ uso da EDG é amplamente sugerido e utilizado dentro de contextos clínicos e de pesquisa. Estudos apontam que a EDG com 15 itens, 
apresenta confiabilidade, validade, sensibilidade e coerência na identificação do transtorno de humor depressivo na população idosa. ${ }^{26}$

O estudo teve aprovação do Comitê de Ética em Pesquisa UniFIEO sob o número 853.742. Todos os indivíduos concordaram em participar voluntariamente mediante a compreensão e assinatura do Termo de Consentimento Livre e Esclarecido (TCLE). Posteriormente foram apresentados aos instrumentos de avaliação, respectivamente o Mini-exame do Estado Mental (MEEM) e a Escala de Depressão Geriátrica (EDG) abreviada com 15 itens, e ainda responderam um questionário semiestruturado com perguntas sociodemográficas e relativas a autonomia e independência nos últimos 30 dias. O tempo médio de aplicação foi de 40 minutos. Seguindo o plano de análise de dados, foram realizadas análises estatísticas descritivas e inferenciais dos resultados obtidos por meio dos instrumentos de avaliação, com o objetivo de verificar o desempenho dos participantes. As analises inferências objetivaram identificar possíveis relações entre os instrumentos de rastreio.

\section{RESULTADOS E DISCUSSÃO}

Os resultados obtidos por meio do instrumento MEEM, demonstraram que dentre os 20 participantes, 11 (55\%) apresentaram comprometimentos cognitivos e $9(45 \%)$ não apresentaram alterações significativas neste constructo. Seguindo tal resultado, a amostra foi dividida em 2 grupos, ou seja, grupo SC (sem comprometimento cognitivo) e grupo CC (com comprometimento cognitivo). A média de idade do grupo SC correspondeu a 70,6 anos e o grupo CC correspondeu a 67,6 anos de idade. A Tabela 1, demonstra os resultados de ambos os grupos nos instrumentos MEEM e EDG.

Tabela 1. Resultados aferidos nos instrumentos MEEM e EDG. Grupo CC (com comprometimento cognitivo) e grupo SC (sem comprometimento cognitivo).

\begin{tabular}{ccccc}
\hline Instrumento & Grupo & Média & Desvio Padrão & Ponto de corte (EDG) \\
\hline MEEM & SC & 28,8 & 1,2 & \\
& CC & 24,9 & 2,1 & $>5$ \\
\hline \multirow{2}{*}{ EDG } & SC & 4,0 & 2,6 & $>5$ \\
\hline
\end{tabular}

Estudos anteriormente realizados não entram em concordância enquanto a taxa de prevalência de déficits cognitivos em pessoas idosas, o intervalo encontrado entre artigos variou de $18,7 \%$ a 46\%. ${ }^{27-28-29-30}$ Tal controvérsia remete a diferentes fatores metodológicos 
relacionados as especificidades das amostras, como as variáveis territoriais, contexto social, diferenças sociodemográficas, presença de comorbidade, entre outras.

É observável na Tabela 2, que indivíduos com baixo nível de escolaridade formal apresentam menores níveis parciais de preservação cognitiva. Ao considerar a nota de corte do instrumento MEEM, fez-se possível indicar o déficit cognitivo do grupo CC pormenorizada na categoria escolaridade, indivíduos com 1 a 4 anos de estudo obtiveram 2,5 pontos abaixo do esperado e sujeitos com formação acima de 11 anos apresentaram déficit de 3,4 pontos. Neste estudo a escolaridade não esteve associada a níveis superiores de preservação cognitiva, tal resultado diverge da literatura cientifica. ${ }^{10,31,27}$ No entanto, pessoas com comprometimento cognitivo de maior escolaridade tiveram níveis de cognição semelhantes ao de idosos com escolaridade baixa, o que tende a ser um dado consonante com a literatura no que se refere à reserva cognitiva. Há que se considerar as características do grupo especificamente, já que o $\mathrm{N}$ pequeno de pessoas por grupo também impacta este tipo de análise e é uma limitação específica deste estudo.

Tabela 2. Distribuição da variável escolaridade dos grupos SC e CC.

\begin{tabular}{cccccc}
\hline Variável & Categoria & Grupo & $\mathbf{n}(\%)$ & Média MEEM & $\begin{array}{c}\text { Desvio } \\
\text { Padrão }\end{array}$ \\
\hline Escolaridade & 1 a 4 anos & SC & $1(33 \%)$ & 26 & - \\
& \multirow{3}{*}{9 a 11 anos } & CC & $2(67 \%)$ & 22,5 & 2,1 \\
& \multirow{3}{*}{11 anos +} & SC & $3(75 \%)$ & 29 & 1,0 \\
& & CC & $1(25 \%)$ & 24 & - \\
& SC & $5(38 \%)$ & 29,2 & 0,45 \\
& CC & $8(62 \%)$ & 25,6 & 1,85 \\
\hline
\end{tabular}

A presença de sintomas depressivos com investigada pela EDG foi encontrada em $30 \%(n=6)$ da amostra. Há estudos na literatura cientifica que identificaram a prevalência similar de sintomas depressivos em indivíduos idosos. ${ }^{32}$ Outros estudos frequentemente encontram indicadores de sintomas depressivos em índices superiores a $25 \%$ das populações longevas investigadas. ${ }^{33-34-35}$

Ao analisarmos particularmente a parcela da amostra com sintomalogia depressiva, como é possível observar na Tabela 3, encontramos variação entre os indicadores de ambos os grupos. Dentre os participantes com sintomalogia depressiva rastreada, 67\% eram do grupo SC e 33\% do grupo CC. Não obstante, a quantidade de sintomas depressivos no grupo CC foi $30 \%$ superior quando comparado ao grupo SC, ou seja, tal 
resultado indica a relação entre decréscimo cognitivo e a maior presença de sintomas depressivos em indivíduos idosos. A presença elevada de sintomas depressivos indica comprometimento funcional em atividades cotidianas. ${ }^{36}$ Especificamente na depressão geriátrica, há importantes alterações nas funções executivas e na memória, sendo estas duas habilidades fundamentais para a qualidade de vida, independência e autonomia dos indivíduos. ${ }^{37}$

Dentre os fatores que mais comprometem a qualidade de vida da população idosa estão os fatores psiquiátricos de depressão e demência. ${ }^{38} \mathrm{~A}$ presença de sintomas depressivos está associada de maneira bilateral a funcionalidade dos indivíduos idosos, a presença de sintomas depressivos causa alterações cognitivas e funcionais, similarmente, dificuldades funcionais prévias aumentam a vulnerabilidade no desenvolvimento de quadros depressivos. ${ }^{39}$ De fato, os dados observados estão em consonância com os da literatura já que o comprometimento cognitivo relacionou-se a maiores sintomatologias depressivas.

Tabela 3. Resultados da EDG referente a parcela da amostra com sintomas depressivos dos grupos CC e SC.

\begin{tabular}{ccccc}
\hline Instrumento & Grupo & Média & $\begin{array}{c}\text { Desvio } \\
\text { Padrão }\end{array}$ & $\begin{array}{c}\text { Ponto de corte } \\
\text { (EDG) }\end{array}$ \\
\hline EDG & SC & 6,3 & 1,9 & $>5$ \\
& CC & 9 & 1,4 & $>5$ \\
\hline
\end{tabular}

Por meio das entrevistas sobre os âmbitos de independência e autonomia da amostra, foi observado que todos os participantes responderam de formas negativas, relatando que a dependência é relativa a sentimentos de incapacidade e desânimo, em vista da possibilidade de não conseguirem realizar aquilo que desejam sem auxílio. Em relação aos últimos 30 dias, os indivíduos perceberam diminuição de suas capacidades gerais. Este dado revela que os indivíduos tem a percepção gradual de suas dificuldades de vida diária. A apercepção subjetiva da capacidade funcional e cognitiva está associada ao desempenho objetivo deste ${ }^{40}$ a diminuição nas capacidades gerais remete ao fato que as escolhas individuais pautadas na autonomia e independência ficam comprometidas cotidianamente.

A qualidade da autonomia e independência nos indivíduos longevos podem ser fatores antagônicos ou de suporte para a qualidade de vida, antagônicos já que quando negativos implicam em incapacidade, inatividade e dependência, porém quando positivos apresentam suporte por meio de participação, independência e integração. ${ }^{41}$ Considerando as 
implicações funcionais relacionadas a déficits cognitivos, há compreensão que a presença de possíveis sintomas depressivos contribui para maior vulnerabilidade desses domínios. A fragilidade das funções executivas devido a quadros depressivos ${ }^{37}$ expressa implicações na independência e autonomia da população idosa. As funções executivas consistem em processos cognitivos que possibilitam a volição, planejamento, ação assertiva e a verificação desse processo. ${ }^{41}$ Sendo assim, déficits em tais funções remete ao comprometimento nas capacidades de autonomia e independência, dois fatores necessários para tomadas de decisões assertivas, qualidade de vida e bem-estar dos indivíduos idosos.

\section{CONSIDERAÇÓES FINAIS}

O indivíduo é considerado saudável ao manifestar dois conceitos significativos para a qualidade de vida, sendo eles a autonomia e a independência. Atentando a importância de tais características na dinamicidade da pessoa idosa, o presente estudo teve como objetivo indicar que aspectos cognitivos e de sintomas depressivos tendem a estar relacionados ou imbricados em pessoas idosas, no âmbito de sua autonomia e independência. Os métodos empregados nesta pesquisa viabilizaram atender o objetivo proposto, por meio do estudo transversal e com apoio da literatura cientifica.

A partir dos resultados apresentados aventa-se que o declínio nas capacidades cognitivas, observadas por meio das avaliações realizadas, implica na dificuldade do indivíduo em manifestar sua autonomia, especialmente na realização de atividades diárias, contribuindo com implicações em sua independência e bem-estar. A capacidade em desempenhar atividades instrumentais básicas é considerada uma predita positiva na qualidade de vida dos idosos, com o declínio da capacidade funcional tal preditor se torna uma implicação negativa, indicando maiores problemas e dificuldades para tal grupo.

Em vista dos resultados descritos, os participantes verbalizaram percepções negativas com sentimentos de desajustes e sofrimento psíquico e social perante a perda de autonomia e independência, faz-se necessário a incitação que a deterioração em tais aspectos, tendem a dificultar diversas dimensões do bem-estar individual. Tal fato, sugere que com a consciência da diminuição de habilidades funcionais há maior sentimento de limitação e por consequência piores prognósticos. A partir disso, faz-se importante preconizar para que profissionais da saúde busquem estratégias clinicas efetivas que 
preservem a autonomia e a independência dos indivíduos idosos, preservando desta maneira a qualidade de vida.

O rastreio cognitivo relacionado ao de sintomas depressivos, indica que indivíduos com alterações cognitivas tendem a apresentar maior quantidade de sintomas depressivos. Salienta-se que mudanças no constructo cognitivo como observados em funções executivas, comumente associadas existência de sintomas depressivos, pressupõe dificuldades nas tomadas de decisões, implica negativamente na autonomia do sujeito e nas atividades cotidianas. Neste sentido, é possível concluir que a presença de alterações cognitivas e de sintomas depressivos podem ser indicadores de maior vulnerabilidade sobre a autonomia e independência em pessoas idosas.

Destaca-se como limitação deste estudo o número de participantes e o caráter homogêneo das características sociodemográficas, especificamente a alta escolaridade da amostra, dificultando desta maneira a generalização para a população idosa. Sendo assim, ressalta-se a necessidade de estudos que busquem a compreensão dinâmica da população idosa e fatores que tenderam a impactar nas características de independência e autonomia, buscando desta maneira a criação de políticas preventivas com base em determinadas faixas etárias, bem como sobre a presença de diminuição do aparato cognitivo por menor que seja, uma vez que mesmo sutil, irá comprometer o cotidiano das pessoas.

\section{REFERÉNCIAS}

1. Moraes EN, Moraes FL, Lima SPP. Características biológicas e psicológicas do envelhecimento. Rev. méd. Minas Gerais. 2010; 20(1): 67-73.

2. Kandel ER, Schwartz JH, Jessell TM, Siegelbaum SA, Hudspeth AJ. Princípios de Neurociências. 5. ed. Porto Alegre: AMGH; 2014.

3. Penna FB, Santo FHE. O Movimento das emoções na vida dos idosos: um estudo comum grupo da terceira idade. Rev. eletrônica enferm. 2006; 8(1): 17-24

4. Ferreira OGL, Maciel SC, Silva AO, Santos WS, Moreira MASP. O envelhecimento ativo sob o olhar de idosos funcionalmente independentes. Rev. esc. enferm. USP. 2010; 44(4): 1065-69.

5. Baltes PB. On the incomplete architecture of human ontogeny: Selection, optimization, and compensation as foundation of developmental theory. Am. psychol. 1997; 52(4): 36680.

6. Silva IR. Papéis Sociais e Envelhecimento em uma Perspectiva de Curso de Vida. Psicol. teor. pesqui. 2000; 16(1): 31-40. doi: http://dx.doi.org/10.1590/S0102-37722000000100005 
7. Neri AL. Bem-estar Subjetivo, Personalidade e Saúde na Velhice. In: Freitas EV, Py L, editores. Tratado de Geriatria e Gerontologia. 3. ed. Rio de Janeiro: Guanabara Koogan; 2013. p. 2068-83.

8. Instituto Brasileiro de Geografia e Estatística. Projeção da população do Brasil e das Unidades da Federação. Disponível em: https://www.ibge.gov.br/apps/populacao/projecao/

9. Instituto Brasileiro de Geografia e Estatística. Pesquisa Nacional de Saúde. Disponível em: https://biblioteca.ibge.gov.br/visualizacao/livros/liv91110.pdf

10. Cancino M, Rehbein-Felmer L, Ortiz MS. Funcionamiento cognitivo em adeltos mayores: rol de la reserva cognitiva, apoyo social y depresión. Rev. méd. Chile. 2018; 146(3): 31522. doi: https://dx.doi.org/10.4067/s0034-98872018000300315

11. Organização Mundial da Saúde. Constituição da Organização Mundial da Saúde. Documentos básicos, suplemento da 45. ed. 2006.

12. Lemos N, Medeiros SL. Suporte Social ao Idoso Dependente. In: Freitas EV, Py L, editores. Tratado de Geriatria e Gerontologia. 3. ed. Rio de Janeiro: Guanabara Koogan; 2013. P. 2001-10.

13. Neri AL, Neri ML. Envelhecimento Cognitivo. In: Freitas EV, Py L, editores. Tratado de Geriatria e Gerontologia. 3. ed. Rio de Janeiro: Guanabara Koogan; 2013. p. 2025-83.

14. Sheline YI, Barch DM, Garcia K, Gersing K, Pieper C, Welsh-Bohmer K, et al. Cognitive function in late life depression: relationships to depression severity, cerebrovascular risk factors and processing speed. Biol. psychiatr. 2006; 60(1): 58-65. doi: 10.1016/j.biopsych.2005.09.019

15. Alves CEO, Alves GS, Sudo FK, Lanna ME, Ericeira-Valente L, Moreira DM, et al. Longterm prognosis of geriatric major depression in relation to cognition and white matter integrity: follow up of two cases. J. bras. psiquiatr. 2012; 61(2): 107-13. doi: https://doi.org/10.1590/S0047-20852012000200009

16. Delaloye C, Baudois S, Bilbal F, Remound CD, Hofer F, Lamon M, et al. Cognitive Impairment in Late-Onset Depression. Limited to a decrement in information processing resources? Eur. neurol. 2008; 60(3): 149-54. doi: 10.1159/000144086

17. Novaretti TMS, Radanovic M, Nitrini R. Screening for cognitive impairment in late onset depression in a Brazilian sample using the BBRC-edu. Dement. neuropsychol. 2012; 6(2): 85-90. doi: https://doi.org/10.1590/S1980-57642012DN06020004

18. Purcell R, Maruff $P$, Kyrios $M$, Pantelis $C$. Neuropsychological function in young patients with unipolar major depression. Psychol. med. 1997; 27(6), 1277-85. doi: https://doi.org/10.1017/S0033291797005448

19. Garcia A, Passos A, Campo AT, Pinheiro E, Barroso F, Coutinho G, et al. A depressão e o processo de envelhecimento. Ciênc. cogn. 2006; 7(1): 111-21. 
20. Folstein MF, Folstein SE, McHugh PR. "Mini-mental state": A practical method for grading the cognitive state of patients for the clinician. J. psychiatr. res. 1975; 12(3): 18998. doi: https://doi.org/10.1016/0022-3956(75)90026-6

21. Brucki SMD, Nitrini R, Caramelli $P$, Bertolucci PHF, Okamoto IH. Sugestões para o uso do mini-exame do estado mental no Brasil. Arq. Neuro-Psiquiatr. 2003; 61(3B): 777-81. doi: http://dx.doi.org/10.1590/S0004-282X2003000500014

22. Souza JGS, Soares LA, Souza TCS, Pereira AR, Souza AGS. Miniexame do Estado Mental: Capacidade Psicométrica e Formas de Avaliação. Rev. APS. 2014; 17(1): 101-5.

23. Santos CS, Cerchiari EAN, Alvarenga MRM, Faccenda O, Oliveira MAC. Avaliação da Confiabilidade do Mini-exame do estado mental em idosos e associação com variáveis sociodemográficas. Cogitare enferm. 2010; 15(3): 406-12.

24. Yesavage JA, Brink TL, Rose TL, Lum O, Huang V, Adey M, et al. Development and validation of a geriatric depression screening scale: a preliminary report. J. psychiatry. res. 1983; 17(1): 37-49. doi: 10.1016/0022-3956(82)90033-4

25. Almeida OP, Almeida SA. Confiabilidade da versão brasileira da Escala de Depressão em Geriatria (GDS) versão reduzida. Arq. neuro-psiquiatr. 1999; 57(2B): 421-6. doi: http://dx.doi.org/10.1590/S0004-282X1999000300013

26. Castelo MS, Coelho Filho JM, Siqueira Neto JI, Noleto JCS, Lima JWO. Escala de Depressão Geriátrica com quatro itens: um instrumento válido para rastrear depressão em idosos em nível primário de saúde. Geriatr. gerontol. 2007; 1(1): 28-33.

27. Lima MTR, Silva RS, Ramos LR. Fatores associados à sintomatologia depressiva numa coorte urbana de idosos. J. bras. psiquiatr. 2009; 58(1): 1-7. doi: https://doi.org/10.1590/S0047-20852009000100001

28. Holz AW, Nunes BP, Thumé E, Lange C, Facchini LA. Prevalência de déficit cognitivo e fatores associados entre idosos de Bagé, Rio Grande do Sul, Brasil. Rev bras. epidemiol. 2013; 16(4): 880-8. doi: https://doi.org/10.1590/S1415-790X2013000400008

29. Nascimento RAS, Batista RTS, Rocha SV, Vasconcelos LRC. Prevalência e fatores associados ao declínio cognitivo em idosos com baixa condição econômica: estudo MONIDI. J. bras. psiquiatr. 2015; 64(3): 187-92. doi: https://doi.org/10.1590/00472085000000077

30. Machado JC, Ribeiro RCL, Leal PFG, Cotta RMM. Avaliação do declínio cognitivo e sua relação com características socioeconômicas dos idosos em Viçosa-MG. Rev. bras. epidemiol. 2007; 10(4): 592-605. doi: https://doi.org/10.1590/S1415-790X2007000400017

31. Moraes AL, Guimarães LSP, Joanette Y, Parente MAMP, Fonseca RP, Almeida RMM. Effect of aging, education, reading and writing, semantic processing and depression symptoms on verbal fluency. Psicol. reflex. crit. 2013; 26(4): 680-90. doi: https://doi.org/10.1590/S0102-79722013000400008 
32. Oliveira DAAP, Gomes L, Oliveira RF. Prevalência de depressão em idosos que frequentam centros de convivência. Rev. saúde pública. 2006; 40(4): 734-6. doi: https://doi.org/10.1590/S0034-89102006000500026.

33. Ramos GCF, Carneiro JA, Barbosa ATF, Mendonça JMG. Caldeira AP. Prevalência de sintomas depressivos e fatores associados em idosos no norte de Minas Gerais: um estudo de base populacional. J. bras. psiquiatr. 2015; 64(2): 122-31. doi: https://dx.doi.org/10.1590/0047-2085000000067

34. Cohen R, Paskulin LMG, Prieb RGG. Prevalência de sintomas depressivos entre idosos em um serviço de emergência. Rev. bras. geriatr. gerontol. 2015; 18(2): 307-17. doi:https://dx.doi.org/10.1590/1809-9823.2015.14052

35. Maciel ACC, Guerra RO. Prevalência e fatores associados à sintomatologia depressiva em idosos residentes no Nordeste do Brasil. J. bras. psiquiatr. 2006; 55(1): 26-33. doi: https://dx.doi.org/10.1590/S0047-20852006000100004

36. Stahnke DN, Martins RB, Farias RR, Knorst MR, Kanan JHC, Resende TL. Depressive symptoms and functionality in older adults of the Porto Alegre's primary care. Geriatr. gerontol. aging. 2020; 14(1): 22-30. doi: 10.5327/Z2447-212320201900071

37. Ávila R, Bottino CMC. Atualização sobre alterações cognitivas em idosos com síndrome depressiva. Rev. bras. psiquiatr. 2006; 28(4): 316-20. doi: https://doi.org/10.1590/S151644462006005000010

38. Blazer DG. Depression in Late Life: Review and Commentary. J. gerontol. 2003; 58A(3): $249-65$.

39. Rodrigues CYS, Figueiredo PAC, Montes HJQ, Vila MG. Effects of depression on cognition of patients with mild neurocognitive disorder due to possible Alzheimer's disease. Cienc. psicol. 2017; 11(2): 149-54. doi: https://dx.doi.org/10.22235/cp.v11i2.1486

40. Almeida ML, Dalpubel D, Ribeiro EB, Oliveira ESB, Ansai JH, Vale FAC. Subjective cognitive impairment, cognitive disorders and self-perceived health: The importance of the informant. Dement. neuropsychol. 2019; 13(3): 335-42. doi: https://doi.org/10.1590/198057642018dn13-030011

41. Guerra ACLC, Caldas CO. Dificuldade se recompensas no processo de envelhecimento: a percepção do sujeito idoso. Ciênc. saúde colet. 2010; 15(6): 2931-40. doi: https://doi.org/10.1590/S1413-81232010000600031

42. Uehara $\mathrm{E}$, Charchat-Fichman $\mathrm{H}$, Landeira-Fernandez J. Funções executivas: Um retrato integrativo dos principais modelos e teorias desse conceito. Rev. neuropsicol. latinoam. 2013; 5(3): 25-37. 